\title{
Dynamic Response of Rubbing Happening in a Flexible Rotor-Bearing System
}

\author{
J.M. Chen, D.X. Jiang \\ State Key Laboratory of Control and Simulation of Power \\ System and Generation Equipments \\ Department of Thermal Engineering \\ Tsinghua University \\ Beijing, China
}

\author{
T.T. Deng, J.S. Wang, J.L. Zhong, S.B. Wang \\ Guizhou Electric Power Research Institute \\ Guiyang, China
}

\begin{abstract}
To reduce the steam loss and raise the efficiency of the steam turbine, the vapour seals are applied in the inner cylinder of the steam turbine. The small gap may easily results in the rubbing happening in the inner cylinder. Sometimes, the rotor is a flexible rotor. In this paper, dynamic response of rubbing happening in a flexible rotor-bearing system was studied. A simplified flexible rotor-bearing system was constructed, whose rotating speed was between the 1st and 2nd critical rotating speed. Thermal effect was considered in the analysis, i.e. the rubbing introduce heat and result in the thermally induced vibration. The transient thermal bow was treated as a pair of moments functioning on the shaft segment where rubbing happened. Simulation shows the amplitude and phase of vibration would be stable after a long time. The severity of rubbing affect the stabilization time, which could be regarded as a diagnostic feature to identify the severity of rub happening in a flexible rotor-bearing system.
\end{abstract}

Keywords-thermally induced vibration; equivalent thermal moment; stabilization time; rubbing; flexible rotor-bearing system

\section{INTRODUCTION}

Rubbing is one of common faults for rotating machinery, which happens between the rotating component and the static component. In order to reduce the steam loss and raise the efficiency, steam seals are widely used in the steam turbine. The smaller the gap is, the higher the efficiency of heat-topower is. Sometimes the gap is adjusted too small, which easily results in the rubbing happening in the inner cylinder.

Rubbing happening in the inner cylinder causes fluctuation of vibration. What's even worse, the severe rubbing would result in the permanent bow and scrap the rotor, which causes an economic loss and security risks. Therefore, it is very important to acquire the dynamic rule of this kind of rubbing, which is meaningful to the diagnosis of the rubbing fault happening in the inner cylinder.

Rubbing is a complicated physics problem related to rotor dynamics and heat transfer. Ahmad[1] summarized the related phenomena as four aspects including non-linear, fractal and chaos, reverse whirling, stiffening and Newkirk effect. The former 3 phenomena are produced by the impact and friction. Due to the high stiffness of steam turbine rotor, the influence of the impact and friction is too small to be observed. On the contrary, thermal induced vibration would be obvious in the rubbing of large-capacity steam turbine, which is called Newkirk effect.
Newkirk effect was firstly put forward in 1926 only as a phenomenon. Dimarogonas[2] illustrated the mechanism of Newkirk effect, i.e. the vibration fluctuation due to the asymmetric thermal bow caused by rubbing. Three modes of the Newkirk effect were discovered according to the rotating speed: spiralling, oscillating and constant modes. Based on a Jeffcott model, Kellenberger[3] derived the motional equation considering the frictional thermal influence. In the analysis, the direction of thermal bow default to be the opposite direction of the impact force. Childs[4] modified the Kellenberger's model by considering the angle between the direction of thermal bow and that of the impact force. Schmied[5] utilized finite element method to analyse the dynamic response of rubbing in a rotor-disc-bearing system. Spiralling mode was simulated in the analysis. Muszyhnska et al.[6] studied the coupled thermal-mechanical effect in the rubbing. The spiralling vibration induced by the transient thermal bow was simulated. Goldman et al.[7] discussed the timescales in the derivation of coupled thermal-mechanical equations. The temperature field was slowly varying compared with the dynamic response of the rotor system. Therefore, the varying of temperature field could be regarded as a quasi-static problem. Bachschmid et al.[8] modelled 1-dimensional rotor dynamic system and 3-dimensional thermal system. The motion of rotor system was acquired in frequency domain and temperature distribution was solved in time domain respectively.

In this paper, a flexible rotor-bearing system was constructed based on finite element method. The dynamic response of rubbing was simulated. The impact components were the shaft and the stator in the middle of the shaft. Due to the big inertia of the shaft, thermal induced vibration was considered as the main phenomenon. Based on the simulated results, the dynamic characteristics of a flexible rotor-bearing system were analysed via the vibrational changes.

\section{THEORY}

\section{A. Rotor Dynamics Analysis}

Finite element method was applied to construct the model of a flexible rotor-bearing system showed in Figure 1. The object that rubs with the shaft is the seal. 1st critical rotating speed and 2nd critical rotating speed were calculated as 1620 rpm and $5244 \mathrm{rpm}$. The shaft rotates at $3000 \mathrm{rpm}$, which makes it a flexible rotor-bearing system. 


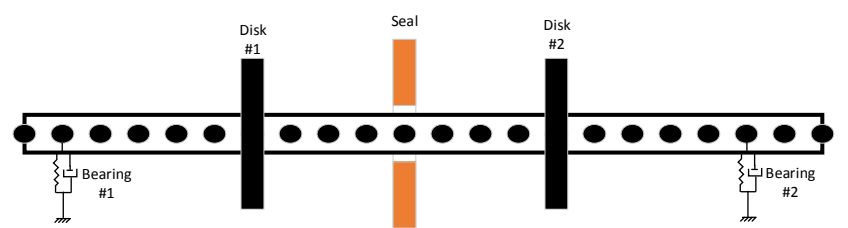

FIGURE I. THE SHAFT CHAIN AND THE RUBBING POSITION

From Figure 1, the flexible rotor-bearing system is simplified as 21 shaft beams, 2 disks and 2 bearings. The motion vector set at every node satisfies the motion equation as follow,

$$
\begin{gathered}
\mathbf{M}\left\{\ddot{\vec{\delta}}_{1}\right\}+(\mathbf{C}+\Omega \mathbf{G})\left\{\dot{\vec{\delta}}_{1}\right\}+\mathbf{K}\left\{\vec{\delta}_{1}\right\}=\left\{\vec{F}_{0}\right\}+\left\{\vec{F}_{\tau}\right\}+\left\{\vec{F}_{n}\right\}+ \\
\left\{\vec{M}_{t h}\right\}
\end{gathered}
$$

where $\left\{\vec{\delta}_{1}\right\}$ is the translational motion vector set at every node, $\mathrm{M}, \mathrm{C}, \mathrm{G}$ and $\mathrm{K}$ are the model matrix, $\Omega$ is the rotational speed, $\left\{\vec{F}_{0}\right\}$ is the harmonic excitation due to unbalance, $\left\{\vec{F}_{\tau}\right\}$ is the frictional force vector due to rubbing, $\left\{\vec{F}_{n}\right\}$ is the normal contact force vector due to rubbing, $\left\{\overrightarrow{\mathrm{M}}_{\text {th }}\right\}$ is the bending moment due to the thermal effect.

In the normal condition, the vibration amplitude of the rotor would not exceed the radius gap and rubbing wouldn't happen. However, if the balance condition is worsened, the rubbing would happen. Figure 2 shows the schematic motion of the rotor and the seal, where 0 is the original point in the absolute coordinate system. $\mathrm{O}_{1}$ is the center of the shaft at the rubbing plane, $\mathrm{O}_{2}$ is the centre of the seals, $\mathrm{P}_{1}$ and $\mathrm{P}_{2}$ are respectively rubbing points in the rotor surface and the seal. $\vec{\delta}_{1, \mathrm{p}}$ is the displacement vector of the rotor center at the rubbing plane. $\vec{\delta}_{2}$ is the displacement vector of the rub-impact seal. As the seal is light and it could make backward motion, which satisfies

$$
\left|\vec{\delta}_{2}\right|=\left\{\begin{array}{cc}
0 \quad, & \text { 当 }\left|\vec{\delta}_{1, p}\right| \leq \varepsilon \\
\left|\vec{\delta}_{1, p}\right|-\varepsilon, & \text { 当 }\left|\vec{\delta}_{1, p}\right|>\varepsilon
\end{array}\right.
$$

where $\varepsilon$ is the radius gap.

As the seal is functioned by the preload of the leaf spring, the contact force satisfies,

$$
\begin{aligned}
& \vec{F}_{n}=\left[k_{s}\left(\left|\vec{\delta}_{1, p}\right|-\varepsilon\right)+F_{\text {in }}\right] \vec{n} \\
& \vec{F}_{\tau}=-\mu_{s}\left|\vec{F}_{n}\right| \vec{\tau}
\end{aligned}
$$

where $\mathrm{k}_{\mathrm{s}}$ is the equivalent stiffness of the leaf spring, $\mathrm{F}_{\text {in }}$ is the preload of the leaf spring, $\mu_{\mathrm{s}}$ is the frictional coefficient.

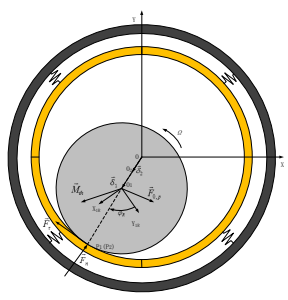

FIGURE II. SCHEMATIC MOTION OF THE ROTOR AND THE STEAM SEALS

\section{B. Thermal Analysis}

Thermal effect caused by rubbing generates the frictional heat, which results in temperature rise in part of the rotor. The bending moment $\overrightarrow{\mathrm{M}}_{\mathrm{th}}\left(\mathrm{M}_{\mathrm{thx}}, \mathrm{M}_{\mathrm{thy}}\right)$ satisfies,

$$
\begin{aligned}
& M_{t h x}=\int_{A} \gamma_{0} E t(x, y) y d A \\
& M_{t h y}=\int_{A} \gamma_{0} E t(x, y) x d A
\end{aligned}
$$

where $\gamma_{0}$ is the linear expansion coefficient, $\mathrm{E}$ is the elastic module and $t(x, y)$ is the temperature at the coordinates $(x, y)$.

From Equation (4), in order to acquire the bending moment $\left\{\overrightarrow{\mathrm{M}}_{\mathrm{th}}\right\}$, the transient temperature field is supposed to be calculated, which is a heat transfer problem. In this analysis, axial heat conduction was ignored. A 2-dimensional model where rubbing happened was utilized. The temperature at each node satisfies,

$$
\begin{aligned}
\rho c \frac{\partial}{\partial \tau}\{t\}= & \frac{\partial}{\partial x}\left(\lambda \frac{\partial}{\partial x}\{t\}\right)+\frac{\partial}{\partial y}\left(\lambda \frac{\partial}{\partial y}\{t\}\right)+\{\dot{q}\} \\
& -\left.\lambda \frac{\mathrm{d} t}{\mathrm{~d} n}\right|_{w}=h\left(t-t_{\infty}\right)
\end{aligned}
$$

where $t$ is the temperature, $\rho c$ is the heat capacity, $\lambda$ is the heat conductivity and $\dot{q}$ is the frictional heat source. $h$ is the convective heat transfer coefficient, $t_{\infty}$ is the temperature of the surroundings.

Based on frictional thermal principle, the frictional heat source could be expressed as

$$
\dot{q}=\kappa\left|\vec{v}_{p 1} \cdot \vec{F}_{\tau}\right|
$$

where $\kappa$ is the ratio of heat that rotor acquires.

\section{Solution Strategy}

The rubbing problem is a coupled structural-thermal problem. In this paper, sequential coupling method is used to simulate the rubbing process. Because of the big inertia of the rotor, rub-induced shock is ignored in the analysis, i.e. only $1 \mathrm{x}$ component of the excitations considered. The flow chart of the calculation program is showed in Figure 3. The heat source is the input of the thermal equation. The thermal bending moment and the contact force are updated in the next time step, which is the input of the rotor dynamics solution. Therefore, the simulation results could be calculated with time approaching.

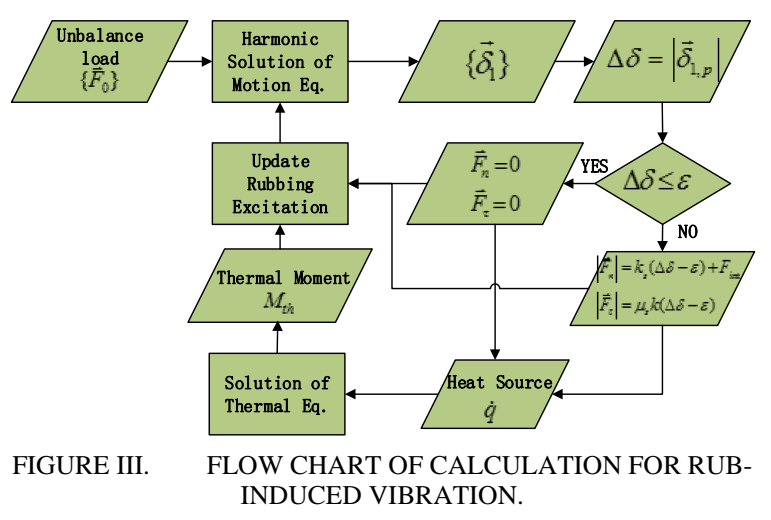




\section{RESULTS AND ANALYSIS}

Light, medium and severe rubs under the working rotating speed were simulated. The difference of the cases was the amplitude of imbalance force. Figure 4 shows the amplitude and phase of $1 \mathrm{x}$ component of the vibration in the rubbing plane. In the normal working condition, the vibration amplitude was within the gap and no contact happened. The vibration phase was nearly $180^{\circ}$ which was the opposite direction of the phase of the imbalance force.

With the balance condition worsened, the amplitude of the vibration rise quickly, which result in rubbing in different severity. Vibration of three cases rise suddenly and then developed into a stable condition. The severe rubbing caused a $0.16 \mathrm{~mm}$ rise and the stabilization time last 203s. However, in the light rubbing the vibration amplitude stayed $0.5 \mathrm{~mm}$, which was the gap value, and the stabilization time was only 178 . The change of the phase was very small in three cases.

The changes of dynamic response were produced by the thermal bending moment. The magnitude of the thermal bending moment was showed in Figure 5. In the light rubbing, the magnitude of the bending moment was nearly $2.1 \times$ $10^{4} \mathrm{~N} \cdot \mathrm{mm}$, which compensated the abnormal imbalance. Due to the compensation of the thermal effect, the dynamic response of the rotor could recover from the big vibration. The self-balance phenomenon was because of the inverse phase of the vibration when the rotor was working between the 1st critical rotating speed and the 2nd critical rotating speed. The thermal effect makes the rotor-bearing system less risky. However, in the medium and severe rubbing, the thermal bending moment could not fully compensate the imbalance in spite of self-balance.

The temperature of the surface rise quickly while rubbing happened, seen as Figure 6. After a period of time, the temperature field become stable. The fluctuation in the light rubbing was caused by the time step length.

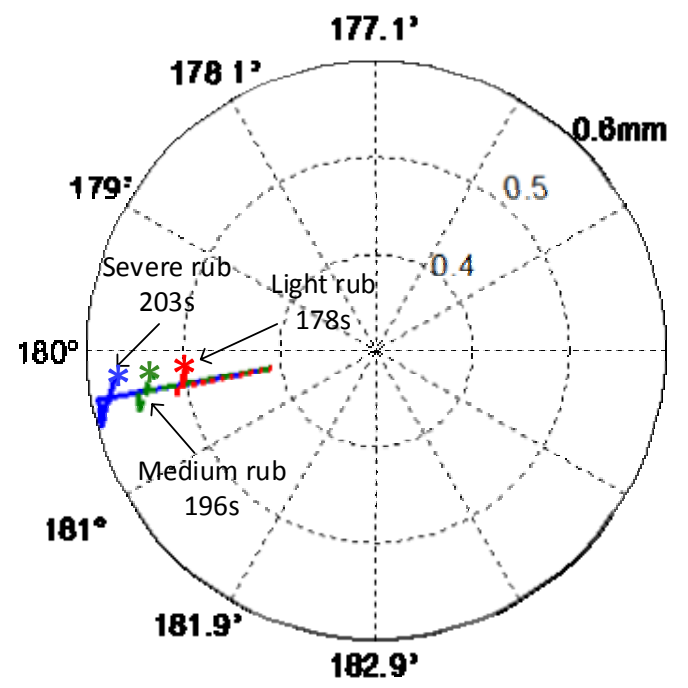

FIGURE IV. AMPLITUDE AND PHASE OF 1X COMPONENT OF THE VIBRATION AT THE RUBBING POSITION

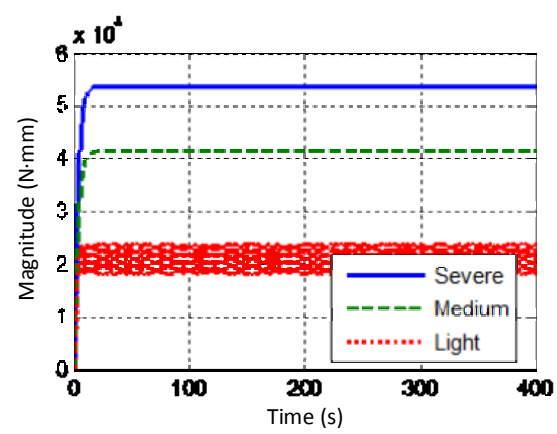

FIGURE V. MAGNITUDE OF THE THERMAL BENDING MOMENT.

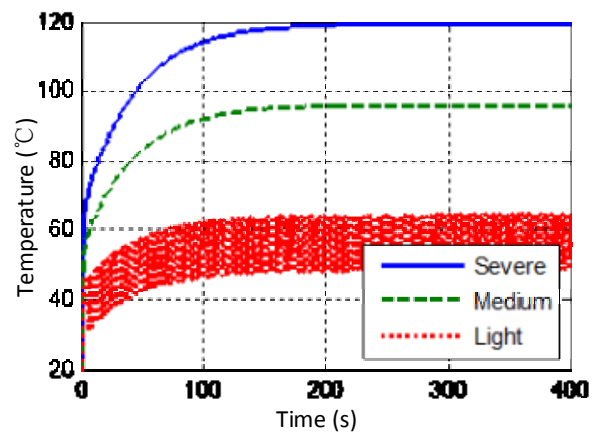

FIGURE V. MAXIMUM OF TEMPERATURE OF THE ROTOR PLANE

\section{CONCLUSION}

Rubbing is a common but annoying fault in the operation of the steam turbine, which could not only result in the abnormal vibration but also do the damage to the safety of the power system. The rubbing happening in a flexible rotor has its own characteristics.

In the paper, the dynamic response of the rubbing happening in a flexible rotor-bearing system was analysed. The rotor dynamics model was constructed based finite element method. The rubbing position located in the middle of the shaft. As thermal effect was considered as the main impact factor, the sequential coupled structural thermal method was applied to solve the coupled problem. Simulation results showed that the vibration of the rotor could develop into a stable mode while the rotating speed was between the $1^{\text {st }}$ critical rotating speed and $2^{\text {nd }}$ critical rotating speed, which was called self-balance phenomenon. The severity of the rubbing would influence the stabilization time, which could be regarded as a diagnostic feature.

\section{REFERENCES}

[1] Ahmad, S., Rotor casing contact phenomenon in rotor dynamicsliterature survey. Journal of Vibration and Control, 16(9): pp. 1369 1377, 2010.

[2] Dimarogonas, A.D., Newkirk effect: thermally induced dynamic instability of high-speed rotors. American Society of Mechanical Engineers (Paper), (73 -GT-26), 1973.

[3] Kellenberger, W., Spiral vibrations due to the seal rings in turbogenerators thermally induced interaction between rotor and stator. Journal of Mechanical Design, 102(1): pp. 177 - 184, 1980. 
[4] Childs, D.W., A note on Kellenberger's model for spiral vibrations. Journal of Vibration and Acoustics, 123(3): pp. 405 - 408, 2001.

[5] Schmied, J., Spiral vibrations of rotors. Proceedings of the ASME Design Technology Conference, Boston, MA, USA, 1987.

[6] Muszyhnska, A., Thermal/mechanical effect of rotor-to-stator rubs in rotating machinery. Proceedings of the 14th Biennial ASME Design Technical Conference on Mechanical Vibration and Noise, Albuquerque, NM, USA, 1993.

[7] Goldman, P. and Muszynska A., Rotor-to-stator, rub-related, thermal/mechanical effects in rotating machinery. Chaos, Solitons and Fractals, 5(9): pp. 1579 - 1579, 1995.

[8] Bachschmid, N., Pennacchi, P. and Vania, A., Thermally induced vibrations due to rub in real rotors. Journal of Sound and Vibration, 299(4-5): pp. 683-719, 2007. 